\title{
Thresholdless crescent waves in an elliptical ring
}

\author{
Kuan-Hsien Kuo, ${ }^{1}$ YuanYao Lin, ${ }^{1, *}$ and Ray-Kuang Lee ${ }^{1,2}$ \\ ${ }^{1}$ Institute of Photonics Technologies, National Tsing Hua University, Hsinchu 300 Taiwan \\ ${ }^{2}$ Frontier Research Center on Fundamental and Applied Sciences of Matters, National Tsing-Hua University, \\ Hsinchu 300, Taiwan \\ *Corresponding author: yuyalin@mx.nthu.edu.tw
}

Received November 26, 2012; revised February 8, 2013; accepted February 25, 2013; posted February 26, 2013 (Doc. ID 180654); published March 21, 2013

\begin{abstract}
By introducing symmetry-breaking in geometry, we reveal the existence of thresholdless crescent waves, i.e., nonlinear diffractionless modes pinged to the boundary of a curvature, in an elliptical ring. An effective nonlinear Schrödinger equation along the azimuthal direction is derived by taking the transformation in the curvilinear coordinate of elliptical symmetry, which illustrates the formation of trapping potentials (barriers) along the semi-major (minor) axis. Our results demonstrate an alternative but efficient approach to access optical modes with a variety of inhomogeneous potentials. (C) 2013 Optical Society of America

OCIS codes: $240.4350,190.6135$.
\end{abstract}

Crescent waves are localized modes pinged to a circular boundary with a shape similar to a cresent moon [1]. To form crescent waves, the original proposal is to extend the concept of surface modes for electronic Tamm states localized at the interface separating periodic crystalline lattice and homogeneous vacuum space [2]. With the introduction of concentric rings, nonlinear surface waves form at the edge of guiding structures, where an abrupt termination of the periodic potential happens [3]. Direct observations of optical surface states have been demonstrated in photonic lattice edges [4] and periodic waveguide arrays [5]. With the introduction of metallic structures, strongly confined plasmonic surface modes can also exist at the termination of metal-dielectric metamaterials $[\underline{6}-\underline{8}]$, as the counterpart in electronic Shockley states [9].

To generate crescent waves, a guiding potential in the shape of a single ring is enough to support modes pinged to the boundary of a curvature. Such self-trapped nonlinear waves, bifurcating from the linear modes, exist above a certain threshold power. In this Letter, we introduce symmetry-breaking in geometry by analyzing crescent waves in an elliptical ring, which can be realized experimentally in a microcavity based on vertical cavity surface emission lasers [10]. The formation power for crescent waves along the semi-major axis of an elliptical ring is found to be lower than that along the semi-minor axis. By directly numerical simulations, we show that the thresholdless crescent waves can be supported along the semi-major axis when the ellipticity, the ratio between the major and minor axes, is larger than a critical value. Through the curvilinear coordinate transform, the corresponding effective potential along the azimuthal direction is derived, in which the minimum (maximum) values occur just at the semi-major (minor) axis. Without any counterparts in the linear limit, the studies on crescent waves not only provide a step to access optical modes with a variety of microcavities but also serve as a platform for other physical systems, such as Barchan sand dunes made by a unidirectional flow of air.

We consider an optical beam with the complex amplitude $\Psi$, scaled to $1 / \sqrt{n_{2} / 2 n_{0}}$ with the linear and nonlinear refractive indexes denoted by $n_{0}$ and $n_{2}$, respectively, which is propagating along the normalized $z$ axis in a
Kerr-type nonlinear material and described by the generalized nonlinear Schrödinger equation [11]

$$
i \frac{\partial \Psi}{\partial z}+\frac{1}{2}\left(\frac{\partial^{2}}{\partial x^{2}}+\frac{\partial^{2}}{\partial y^{2}}\right) \Psi+|\Psi|^{2} \Psi+V(x, y) \Psi=0
$$

where $x$ and $y$ are transverse coordinates, with the scaled length normalized by $1 /\left(n_{0} k_{0}\right)$ and the free space propagation constant of the wave denoted by $k_{0}$. The potential in our consideration, $V(x, y)$, is an elliptical ring described by

$$
V(r)= \begin{cases}V_{0} \cos ^{2}(\pi r), & \frac{1}{2} \leq r \leq \frac{3}{2} \\ 0, & \text { otherwise }\end{cases}
$$

with the depth of potential $V_{0}$ and radial parameter $r^{2}=$ $x^{2} / a^{2}+y^{2} / b^{2}$ shown in Fig. 1 . Given the semi-major and semi-minor axes, $a$ and $b$, respectively, stationary nonlinear solutions are found numerically by substituting the ansatz $\Psi(x, y ; z)=U(x, y) \exp (i \beta z)$ into Eq. (1), for a given propagation constant $\beta$.

Examples of found crescent waves, showing the intensity profiles, $|\Psi|^{2}$, are illustrated in Figs. $2($ a) and $2(b)$, which are pinged to the boundary of a given elliptical ring. Here, the ellipticity, defined as $a / b=1.8$, is chosen, such that $x(y)$ axis corresponds to the major (minor) axis. In contrast to a random localization in the azimuthal direction for the case in a circularly symmetric
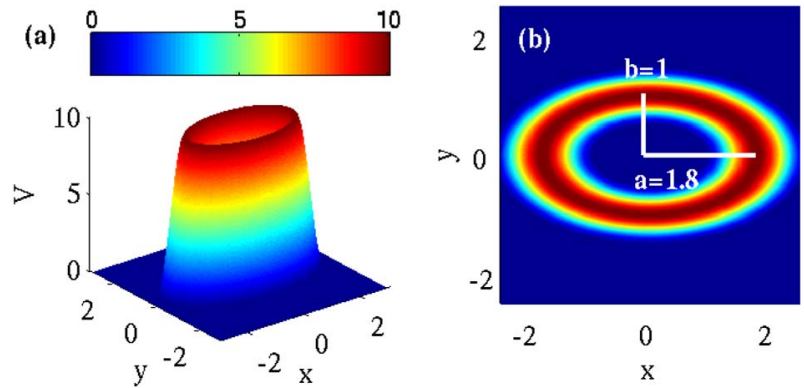

Fig. 1. (Color online) (a) Elliptical potential, $V$, and (b) corresponding top-view used in the simulations, with the semi-major axis $a=1.8$, semi-minor axis $b=1$, and potential depth $V_{0}=10$. 

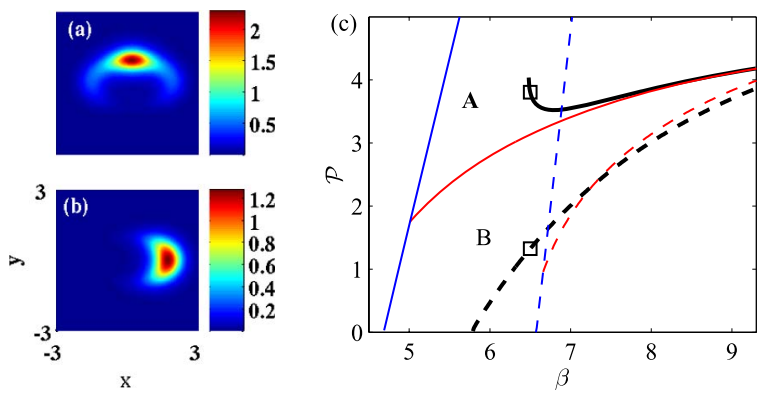

Fig. 2. (Color online) Examples of the intensity profile for crescent waves are shown along the (a) semi-minor and (b) semi-major axes, corresponding to the markers A and B in (c), respectively. (c) Formation power $\mathcal{P}$ versus propagation constant $\beta$ for crescent waves along the semi-minor (solid-line) and semi-major (dashed-line) axes are shown in black, while the crescent waves in the inscribed (dashed-line) and circumscribed (solid-line) circles are shown in red, which bifurcate from the corresponding symmetric donut-shaped modes (in blue).

annular ring [1,12], now the supported crescent waves in an elliptical ring localize only along the minor and major axes. Moreover, no localized solution is found other than the minor and major axes. It can be seen clearly that along the semi-minor axis, the supported crescent wave has an elongated field distribution, almost extending to more than half of the elliptical ring, while along the semi-major axis, the supported one is strongly localized with a narrow field distribution. More interestingly, the required formation powers, defined as $\mathcal{P} \equiv \int_{-\infty}^{\infty}|\Psi|^{2} \mathrm{~d} x \mathrm{~d} y$, are totally different for the crescent waves along the semi-minor and semi-major axes, as shown in Fig. 2(c).

As known for the case in a circular ring, a certain threshold power is needed for nonlinear surface modes to be self-trapped at a random azimuthal orientation [12]. It is also shown in Fig. 2(c) that crescent waves in a circular ring potential bifurcate from the corresponding symmetric donut-shaped modes at some threshold power, as the cases both for inscribed and circumscribed circles shown in blue colors. In the scenario of an elliptical ring, such a threshold power remains for the crescent waves along the semi-minor axis [see the black solid-line in Fig. 2(c)]. Yet along the semi-major axis, as shown in the black dashed-line of Fig. 2(c), the supported crescent waves are thresholdless. Since the threshold power required for a crescent wave comes from its bifurcation from a linear mode, for an elliptical ring, there is no corresponding linear mode to match due to a directly symmetry-breaking in geometry.

By varying the value of ellipticity, in Fig. 3 , we show the corresponding threshold power, $\mathcal{P}_{\text {th }}$, for crescent waves along the $x$ axis (dashed-line). In the case of $a / b=1$, our elliptical ring is reduced into a circular one, which recovers to the known results in the literature [12]. When the value of ellipticity exceeds 1 , the required threshold power for the crescent waves along the semi-major axis decreases. A thresholdless condition is found for those crescent waves in the elliptical ring with the ellipticity above 1.8. On the other side, when the ellipticity goes below 1 , the required threshold power for the crescent waves along the same $(x)$ axis increases. In this case, the original $x$ axis changes from a major axis

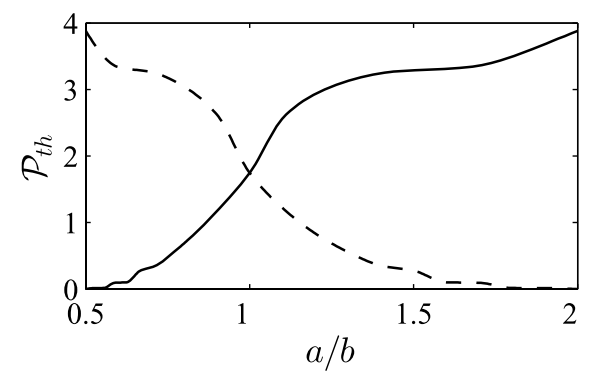

Fig. 3. Required threshold power, $\mathcal{P}_{\text {th }}$, to support crescent waves along the $y$ (solid-line) $/ x$ (dashed-line) axis for different ellipticity, $a / b$.

to a minor one, and thresholdless crescent waves can be found along the $y$ axis, as shown in the solid-line of Fig. 3. The stabilities of these crescent waves are testified both with linear stability analyses by imposing perturbations upon these crescent wave solutions and a direct nonlinear wave propagation numerically. All the crescent waves found are stable due to the confinement from ring potentials. Without the nonlinearity, the crescent waves in an elliptical ring get diffracted in the propagation and eventually flow to the opposite side along the semi-major/semi-minor axis, which is applicable for the implementation of nonlinear switching devices.

To have a better understanding on the formation of crescent waves in an elliptical ring, we transform the nonlinear wave equation in Eq. (1) in the curvilinear coordinate of elliptical symmetry [13], by introducing new variables in the transverse directions, according to the following relation: $x=l \cosh (\mu) \cos (\theta)$ and $y=l \sinh (\mu) \sin (\theta)$. Then the corresponding Laplacian operator in the new coordinate system can be written as

$$
\frac{\partial^{2}}{\partial x^{2}}+\frac{\partial^{2}}{\partial y^{2}}=\frac{1}{h^{2}}\left(\left|\frac{\partial}{\partial \mu}\right|^{2}+\left|\frac{\partial}{\partial \theta}\right|^{2}\right)
$$

where $h=l \sqrt{\cosh ^{2}(\mu)-\cos ^{2}(\theta)}$, with the semi-focal distance, $l$, determined by a given ellipticity. With the variational approach, a separable ansatz $\Psi=$ $\mathcal{M}(\mu) \Theta(\theta) \exp (i \beta z)$ is used as a solitary wave solution for the NLSE with an elliptical ring $[\underline{13}, 14]$. The corresponding Lagrangian density can be described as

$$
\begin{aligned}
\mathcal{L}= & -\frac{1}{2} \frac{1}{h^{2}}\left(\left|\frac{\partial}{\partial \mu} \mathcal{M}\right|^{2}|\Theta|^{2}+|\mathcal{M}|^{2}\left|\frac{\partial}{\partial \theta} \Theta\right|^{2}\right)-\beta|\mathcal{M} \Theta|^{2} \\
& +\frac{1}{2}|\mathcal{M} \Theta|^{4}+V|\mathcal{M} \Theta|^{2} .
\end{aligned}
$$

Supposing that the ring potential $V$ is narrow in $\mu$ direction, one can take $\mathcal{M}(\mu)$ as a parameter to minimize the averaged Lagrangian by integrating the Lagrange density over $\mu$. From the averaged Lagragian, we can reach a quasi-one-dimensional equation of motion for the supported soliton wave solution $\Theta(\theta)$ in the azimuthal direction, i.e.,

$$
\beta \Theta=\frac{\partial}{\partial \theta}\left\{D_{\text {eff }} \frac{\partial \Theta}{\partial \theta}\right\}+g_{\text {eff }}|\Theta|^{2} \Theta+V_{\text {eff }} \Theta,
$$



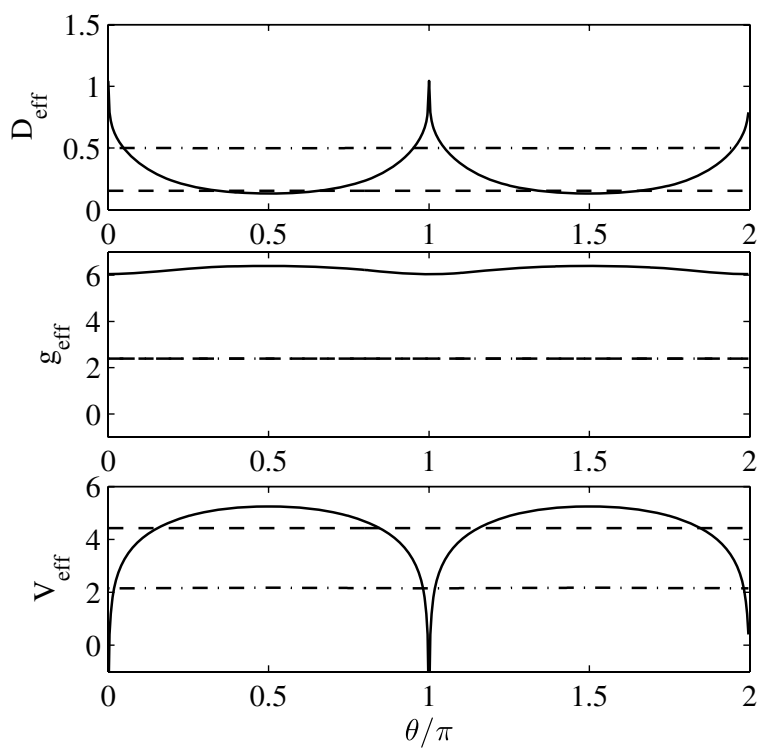

Fig. 4. Effective diffraction coefficient $D_{\text {eff }}$, effective nonlinearity $g_{\text {eff }}$, and effective potential $V_{\text {eff }}$ for the reduced quasi1D nonlinear equation along the azimuthal direction $\theta$, with the formula in Eq. (4). Dashed and dotted-dashed lines in each panel indicate the cases of the inscribed and circumscribed circles, with the radii 1 and 1.8 , respectively.

where the corresponding effective diffraction coefficient $D_{\text {eff }}$, effective nonlinearity $g_{\text {eff }}$, and effective potential $V_{\text {eff }}$ are shown in Fig. $\underline{4}$, with the form given below:

$$
\begin{aligned}
D_{\text {eff }} & =\frac{1}{2} \frac{\int_{0}^{\infty} \frac{1}{h}|\mathcal{M}|^{2} \mathrm{~d} \mu}{2 \int_{0}^{\infty} h|\mathcal{M}|^{2} \mathrm{~d} \mu}, \quad g_{\text {eff }}=\frac{\int_{0}^{\infty} h|\mathcal{M}|^{4} \mathrm{~d} \mu}{\int_{0}^{\infty} h|\mathcal{M}|^{2} \mathrm{~d} \mu}, \\
V_{\text {eff }} & =\frac{-\int_{0}^{\infty} \frac{1}{h}|\mathcal{M} \mu|^{2} \mathrm{~d} \mu+2 \int_{0}^{\infty} h V|\mathcal{M}|^{2} \mathrm{~d} \mu}{2 \int_{0}^{\infty} h|\mathcal{M}|^{2} \mathrm{~d} \mu} .
\end{aligned}
$$

Here the ring potential $V$ is taken from the same parameters in Fig. 1 . From Fig. $\underline{4}$, it is clearly seen that the effective potential energy has maximum values at $\theta=\pi / 2$ and $3 \pi / 2$, which acts as a potential barrier and sets the lower limit (threshold value) on the propagation constant $\beta$ for a localized state to be supported along the semi-minor axis. Instead, the effective potential shows minimum values along the semi-major axis, $\theta=0, \pi$, and $2 \pi$, resulting in the reduction of formation power for crescent wave in a trapping potential, where discontinuity occurs [15]. Compared to the circular ring potentials, no matter the inscribed or circumscribed circles, the effective diffraction coefficient, effective nonlinearity and effective potentials are all constants in the azimuthal directions.

In conclusion, by introducing a potential in shape of elliptical rings, we reveal the existence of two different families of crescent waves along the semi-major and semi-minor axes. For the crescent wave along the semi-major axis, its profile is strongly localized due to the confinement induced by the symmetry-breaking in geometry, resulting in the reduction of formation power to self-support. Thresholdless crescent waves are found numerically and illustrated analytically when the ellipticity of the ring is larger than a critical value. Our studies provide an interesting result for the understanding in the interplay between symmetry-breaking phenomenon and nonlinear modes.

\section{References}

1. C. P. Jisha, Y. Y. Lin, T.-D. Lee, and R.-K. Lee, Phys. Rev. Lett. 107, 183902 (2011).

2. I. E. Tamm, Z. Phys. 76, 849 (1932).

3. Y. V. Kartashov, V. A. Vysloukh, and L. Torner, Opt. Lett. 32, 2948 (2007).

4. S. Suntsov, K. G. Makris, D. N. Christodoulides, G. I. Stegeman, A. Hache, R. Morandotti, H. Yang, G. Salamo, and M. Sorel, Phys. Rev. Lett. 96, 063901 (2006).

5. A. Szameit, I. L. Garanovich, M. Heinrich, A. A. Sukhorukov, F. Dreisow, T. Pertsch, S. Nolte, A. Tunnermann, and Yu. S. Kivshar, Phys. Rev. Lett. 101, 203902 (2008).

6. S. M. Vukovic, I. V. Shadrivov, and Yu. S. Kivshar, Appl. Phys. Lett. 95, 041902 (2009).

7. S. H. Nam, E. Ulin-Avila, G. Bartal, and X. Zhang, Opt. Lett. 35, 1847 (2010).

8. Y. Y. Lin, R.-K. Lee, and Yu. S. Kivshar, Opt. Lett. 34, 2982 (2009).

9. W. Shockley, Phys. Rev. 56, 317 (1939).

10. W.-X. Yang, Y. Y. Lin, T.-D. Lee, R.-K. Lee, and Yu. S. Kivshar, Opt. Lett. 35, 3207 (2010).

11. Yu. S. Kivshar and G. P. Agrawal, Optical Solitons (Academic, 2003).

12. Y. V. Kartashov, V. A. Vysloukh, and L. Torner, Opt. Lett. 34, 283 (2009).

13. Y. Y. Lin and R.-K. Lee, Opt. Lett. 33, 1377 (2008).

14. A. S. Desyatnikov, A. A. Sukhorukov, and Yu. S. Kivshar, Phys. Rev. Lett. 95, 203904 (2005).

15. Y. V. Bludov, Y. V. Kartashov, and V. V. Konotop, Opt. Lett. 35, 3339 (2010). 